\title{
Computational Modeling of Quasi-Periodic Rudin- Shapiro Multilayered Band Gap Structure
}

\author{
Yassine Bouazzi \\ Industrial Engineering Department \\ University of Hail \\ Hail, Saudi Arabia \\ yassine.bouazzi@enit.utm.tn
}

\author{
Naim Ben Ali \\ Industrial Engineering Department \\ University of Hail \\ Hail, Saudi Arabia \\ naimgi2@yahoo.fr
}

\author{
Youssef Trabelsi \\ Physics Department \\ King Khalid University \\ Aseer, Saudi Arabia \\ youssef trabelsi@yahoo.fr
}

\author{
Haitham Alsaif \\ Electrical Engineering Department \\ University of Hail \\ Hail, Saudi Arabia \\ hal-saif@hotmail.com
}

\author{
Attia Boudjemline \\ Industrial Engineering Department \\ University of Hail \\ Hail, Saudi Arabia \\ a_boudjemline@hotmail.com
}

\author{
Ahmed Torchani \\ Industrial Engineering Department \\ University of Hail \\ Hail, Saudi Arabia \\ tochahm@yahoo.fr
}

\begin{abstract}
The optical transmission spectra proprieties of the onedimensional quasi-periodic multilayered photonic structures according to the Rudin-Shapiro distribution are studied theoretically in this paper by using a theoretical model based on the transfer matrix approach for normal incidence geometry. The influence of the layer number has been studied, i.e. the iteration order of the generating sequence of the quasi-periodic structure on the structure spectral behavior and the width of the Photonic Band Gap (PBG). It was found that the width of the PBG is proportional to the index contrast.
\end{abstract}

Keywords-photonic crystal; Rudin-Shapiro distribution; transfer-matrix method; transmittance; photonic band gap

\section{INTRODUCTION}

Photonic crystals are new, artificially created materials in which the refractive index is periodically modulated in a scale compared to the wavelength of operation [1-4]. In fact, the periodic modulation of the refractive index of such a structure causes the appearance of frequency ranges for which the light cannot propagate [5]. Photonic crystals at the end of the last century became one of the most active research topics in various technological disciplines, e.g. communications, optoelectronics, and optics [6,7]. The simplest form of a photonic crystal is the one-dimensional periodic structure, known as the Bragg mirror. It consists of a stack of alternating layers with low and high refractive index and a thickness in the order of $\lambda_{0} / 4$, where $\lambda_{0}$ is the reference wavelength, i.e. the thicknesses satisfy the Bragg condition. There is currently a need to design photonic materials with broader band gaps and multiband. The first manifestation of this goal was the realization of a new class of artificial crystals, called quasiperiodic photonic crystals [8]. These structures are formed by the stacking of two or more deposited materials according to a predefined recursive inflation rule, so that they can be considered as intermediate systems between an ordinary periodic crystal and random amorphous solids. These quasiperiodic structures have become the subject of intense research. They have led to many technological achievements in the fields of photonics, telecommunications, and microwaves [9].

This paper aims to study the optical properties of the photonic quasi-periodic unidimensional multilayer structures from their spectral response using theoretical modeling and numerical simulation based on the Transfer Matrix Method (TMM).

\section{MODELING PROCEDURE AND NUMERICAL SIMULATION}

\section{A. Structure Presentation}

The Rudin-Shapiro sequence, also known as the GolayRudin-Shapiro sequence, is an infinite automatic sequence [10, 11] introduced in 1950 [12]. Rudin-Shapiro's 1D multilayered distribution system was exploited for the first time to study light confinement in multilayer structures in 2008 [13]. The Rudin-Shapiro sequence can be defined by [14-15]:

$$
R S(n)=\varepsilon_{0} \varepsilon_{1} \varepsilon_{2} \ldots \varepsilon_{i} \ldots \varepsilon_{n-1} \varepsilon_{n} .
$$

with:

$$
\varepsilon_{i}=(-1)^{u(i)}
$$

where $u(i)$ represents the number " 11 " in the binary development of the integer $i$.

The use the Rudin-Shapiro distribution in the photonics field remains very limited due to the construction peculiarity of this structure. It is represented by quaternary elementary layers (A, B, C and D) with substitution rules given by:

$$
\left\{\begin{array}{c}
A s=\{A, B, C, D\} \\
A \rightarrow A B, B \rightarrow A C, C \rightarrow D B, D \rightarrow D C
\end{array}\right.
$$


The generation starts with the element labeled by the letter A. For example, a multilayer structure of iteration order 2 is given by: $\mathrm{S}_{2} \mathrm{ACAB}$, order 3 by $\mathrm{S}_{3} \mathrm{ACABACDC} \ldots$ (Figure 1). Table I presents the generation of the structure of iteration order $\mathrm{k}=1,2,3$.

TABLE I. THE FIRST GENERATIONS OF THE 1D MULTILAYER STRUCTURE ACCORDING TO THE RUDIN-SHAPIRO DISTRIBUTION

\begin{tabular}{|c|c|}
\hline Iteration order & Alphabetic sequence \\
\hline $\mathrm{RS}(0)$ & $\mathrm{A}$ \\
\hline $\mathrm{RS}(1)$ & $\mathrm{AC}$ \\
\hline $\mathrm{RS}(2)$ & $\mathrm{ACAB}$ \\
\hline $\mathrm{RS}(3)$ & $\mathrm{ACABACDC}$ \\
\hline
\end{tabular}

In this work, we chose a multilayer photonic system composed of four dielectric layers A, B, C and D, distributed according to the Rudin-Shapiro sequence, having refraction indices $n_{\mathrm{A}}, n_{\mathrm{B}}, n_{\mathrm{C}}$ and $n_{\mathrm{D}}$, and optical thicknesses $\lambda_{0} / n_{\mathrm{A}}$, $\lambda_{0} / n_{\mathrm{B}}, \lambda_{0} / n_{\mathrm{C}}$ and $\lambda_{0} / \mathrm{n}_{\mathrm{D}}$ respectively, where $\lambda_{0}$ is the reference wavelength. Furthermore, the optical and geometrical parameters of the structure obey the quarter wavelength condition or Bragg condition as indicated by (4):

$$
n_{\mathrm{A}} d_{\mathrm{A}}=n_{\mathrm{B}} d_{\mathrm{B}}=n_{\mathrm{C}} d_{\mathrm{C}}=n_{\mathrm{D}} d_{\mathrm{D}}=\frac{\lambda_{0}}{4}
$$

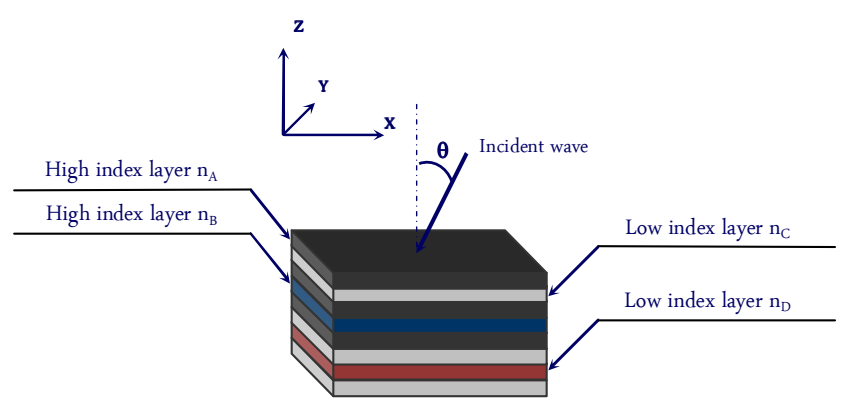

Fig. 1. One-dimensional Rudin-Shapiro multilayer dielectric structure for the $3^{\text {rd }}$ iteration $\operatorname{RS}(3)$ : ACABACDC

\section{B. Simulation Method}

For the calculation of reflection and transmission, we used TMM, which is a technique well suited to the study of PBG materials. This technique can solve the standard problem of the photonic band structures to find transmission, reflection, and absorption spectra. This method consists in expressing the reflected field $\vec{E}_{0}^{-}$from a multilayer structure as a function of incident fields $\vec{E}_{0}^{+}$in a matrix form. The studied multilayer structure consists of a stack of $m$ layers with different thicknesses $d_{i}$ and refractive indices $n_{i}$, as shown in Figure 2. The amplitudes of the electric fields of incident and transmitted wave are expressed by the following matrix for stratified films within $m$ layers:

$$
\left(\begin{array}{l}
E_{0}^{+} \\
E_{0}^{-}
\end{array}\right)=\prod_{1}^{m+1} C_{j}\left(\begin{array}{c}
E_{m+1}^{+} \\
E_{m+1}^{-}
\end{array}\right)
$$

where $C_{j}$ is the propagation matrix for the $j^{\text {th }}$ layer, given by:

$$
C_{j}=\left(\begin{array}{ll}
\frac{1}{t_{j}} \exp \left(-i \varphi_{j-1}\right) & \frac{r_{j}}{t_{j}} \exp \left(i \varphi_{j-1}\right) \\
\frac{r_{j}}{t_{j}} \exp \left(-i \varphi_{j-1}\right) & \frac{1}{t_{j}} \exp \left(-i \varphi_{j-1}\right)
\end{array}\right)
$$

where $\varphi_{\mathrm{j}-1}$ denotes the change in the phase of the wave between $(j-1)^{\text {th }}$ and $j^{\text {th }}$ layers, and can be obtained by:

$$
\left\{\begin{array}{c}
\phi_{0}=0 \\
\phi_{j-1}=\frac{2 \pi}{\lambda} \hat{n}_{j-1} d_{j-1} \cos \theta_{j-1}
\end{array}\right.
$$

$t_{j}$ and $r_{j}$ are the Fresnel transmission and reflection coefficients at the interface between $(j-1)^{\text {th }}$ and $j^{\text {th }}$ layer. The Fresnel coefficients are expressed using the complex refractive index $n_{j}$ and the complex refractive angle $\theta_{j}$ :

For the TM mode, the coefficients are:

$$
\begin{aligned}
r_{j}^{T M} & =\frac{\hat{n}_{j-1} \cos \theta_{j}-\hat{n}_{j} \cos \theta_{j-1}}{\hat{n}_{j-1} \cos \theta_{j}+\hat{n}_{j} \cos \theta_{j-1}} \\
t_{j}^{T M} & =\frac{2 \hat{n}_{j-1} \cos \theta_{j-1}}{\hat{n}_{j-1} \cos \theta_{j}+\hat{n}_{j} \cos \theta_{j-1}}
\end{aligned}
$$

and for the TE mode:

$$
\begin{aligned}
r_{j}^{T E} & =\frac{\hat{n}_{j-1} \cos \theta_{j-1}-\hat{n}_{j} \cos \theta_{j}}{\hat{n}_{j-1} \cos \theta_{j-1}-\hat{n}_{j} \cos \theta_{j}} \\
t_{j}^{T E} & =\frac{\hat{n}_{j-1} \cos \theta_{j-1}}{\hat{n}_{j-1} \cos \theta_{j-1}+\hat{n}_{j} \cos \theta_{j}}
\end{aligned}
$$

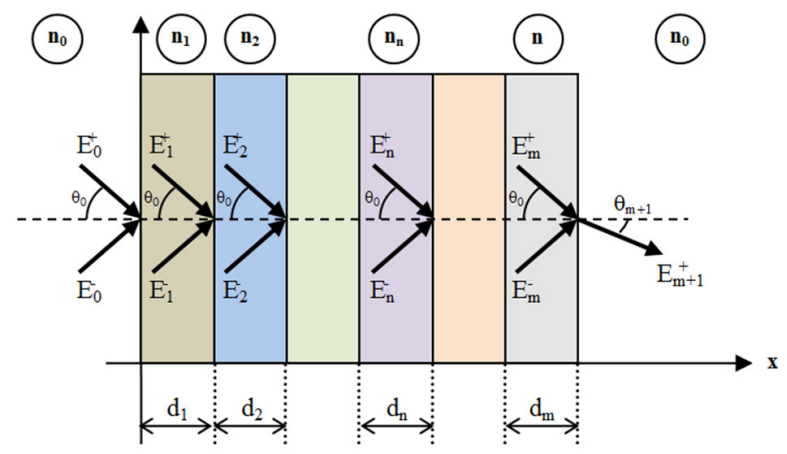

Fig. 2. Multilayer system with $m$ components

Taking $E_{m+1}^{-}=0$, there will be no reflections from the interface of the final structure. Convenient formulas have been obtained for the total reflection and transmission coefficients, which correspond to the amplitude reflectance $r$ and transmittance $t$ respectively, as follows [16-19]:

$$
\begin{aligned}
& r=\frac{T_{21}}{T_{11}} \\
& t=\frac{1}{T_{11}}
\end{aligned}
$$

where $T_{11}$ and $T_{21}$ are the matrix elements of the product matrix:

$$
\prod_{1}^{m+1} C_{j}=\left(\begin{array}{ll}
T_{11} & T_{12} \\
T_{21} & T_{12}
\end{array}\right)
$$

Starting from (12) and (13), the reflection $R$ and the transmission $T$ are given by [6]:

$$
\begin{aligned}
& R=|r|^{2}=\left|\frac{T_{21}}{T_{11}}\right|^{2} \\
& T=|t|^{2}=\left|\frac{1}{T_{11}}\right|^{2}
\end{aligned}
$$




\section{NUMERICAL RESULTS AND DISCUSSION}

Figure 2 shows the reflection spectra with normal incidence for the quasi-periodic multilayer system distributed according to the generalized Rudin-Shapiro model. We choose the following four materials as elementary layers of the stack: $\mathrm{TiO}_{2}$ $\left(n_{\mathrm{A}}=2.3\right), \mathrm{HfO}_{2}\left(n_{\mathrm{B}}=2\right), \mathrm{SiO}_{2}\left(n_{\mathrm{C}}=1.45\right)$ and $\mathrm{TaO}_{5}\left(n_{\mathrm{D}}=2.2\right)$.

(a)

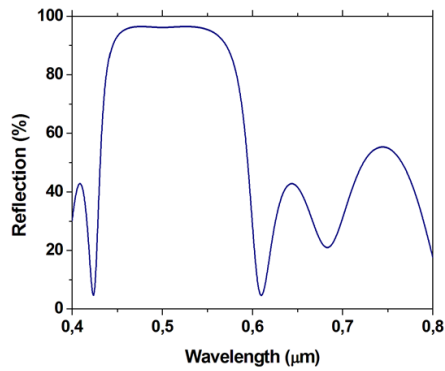

(b)

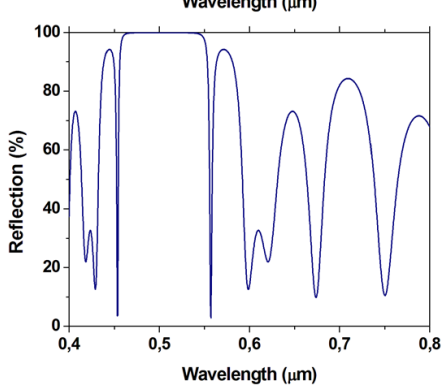

(c)

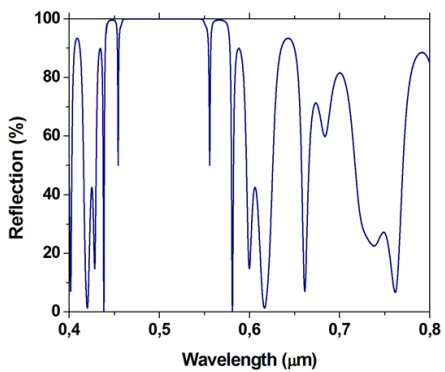

(d)

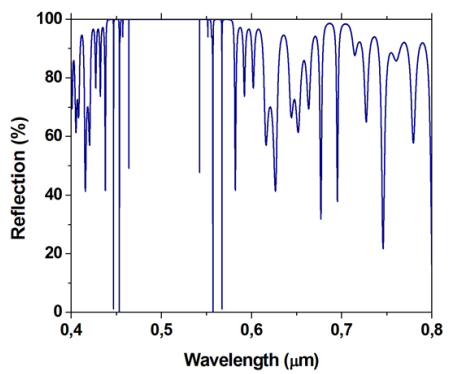

Fig. 3. The reflection spectra of Rudin-Shapiro distribution multilayer photonic structures for: (a) $4^{\text {th }}$, (b) $5^{\text {th }}$, (c) $6^{\text {th }}$, and (d) $7^{\text {th }}$ iteration

We notice from the spectra in Figure 3 that the width of the photonic band gap of the studied system increases with the order of generation $k$. The width of the PBG (Figure 4) observed near $\lambda_{0}=0.5 \mu \mathrm{m}$ increases when progressing from one iteration to another.

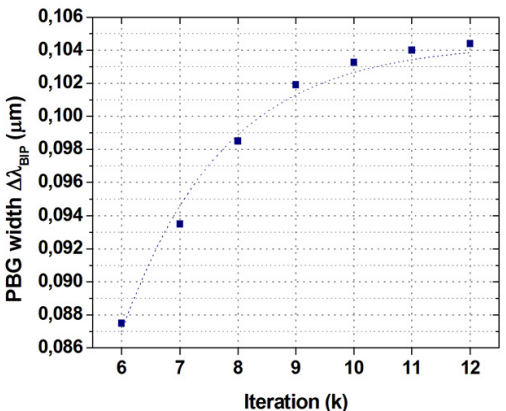

Fig. 4. Variation of the PBG width as a function of the number of iterations $(k)$

TABLE II. WIDTH AND LIMITATIONS OF PBGS

\begin{tabular}{|c|c|}
\hline Iteration $\boldsymbol{k}$ & $\boldsymbol{\Delta} \boldsymbol{\lambda}_{P B G}(\boldsymbol{\mu} \mathbf{m})$ \\
\hline 6 & 0.0898 \\
\hline 7 & 0.0972 \\
\hline 8 & 0.1002 \\
\hline 9 & 0.1025 \\
\hline 10 & 0.1039 \\
\hline 11 & 0.1039 \\
\hline 12 & 0.1039 \\
\hline
\end{tabular}

Figure 5 and Table III show the evolution of the maximum reflection $R_{\max }(\%)$ as a function of the iteration $k$. We can consider that the first PBG appears for the $6^{\text {th }}$ iteration with PBG width $\Delta \lambda_{P B G}=0.1039 \mu \mathrm{m}$.

TABLE III. VARIATION OF $R_{\max }(\%)$ ACCORDING TO $k$

\begin{tabular}{|c|c|}
\hline Iteration k & $\boldsymbol{R}_{\max }(\mathbf{\%})$ \\
\hline 2 & 0 \\
\hline 3 & 29.7051 \\
\hline 4 & 81.2423 \\
\hline 5 & 96.2897 \\
\hline 6 & 99.9874 \\
\hline 7 & 99.9999 \\
\hline 8 & 100 \\
\hline 9 & 100 \\
\hline 10 & 100 \\
\hline
\end{tabular}

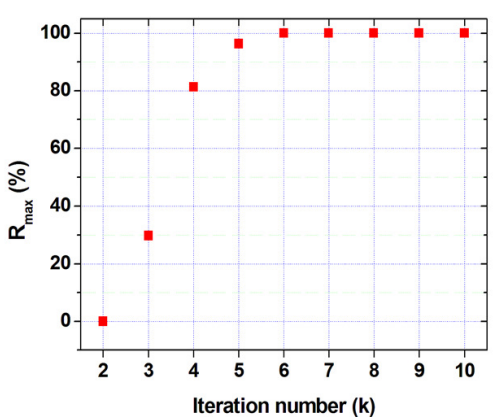

Fig. 5. Variation of $R_{\max }(\%)$ as a function of the iteration value $k$

We notice that starting from the $7^{\text {th }}$ iteration two transparent windows (Figure 6) located in the central PBG and almost symmetrical to $\lambda_{0}=0.5 \mu \mathrm{m}$ with $T=100 \%$ appear. These optical windows indicate a strong light confinement in the microcavities of low refractive indices formed by the quasi- 
periodic stack for the corresponding wavelengths. This last characteristic makes the system a good candidate for the design of an optical filter.

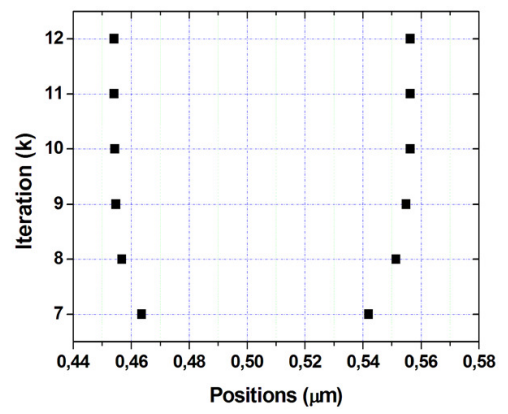

Fig. 6. Number and position of optical windows according to the number of iterations $k$

TABLE IV. WIDTHS OF PBGS, NUMBER OF OPTICAL WINDOWS AND THEIR POSITIONS, ACCORDING TO THE NUMBER OF ITERATIONS $k$

\begin{tabular}{|c|c|c|c|}
\hline \multirow{2}{*}{$\mathbf{k}$} & \multirow{2}{*}{$\Delta \lambda_{P B G}$} & \multicolumn{2}{|c|}{ Optical windows } \\
\cline { 3 - 4 } & & Number & Positions \\
\hline 6 & 0.0898 & - & - \\
\hline 7 & 0.0972 & 2 & $0.4636-0.5421$ \\
\hline 8 & 0.1002 & 2 & $0.4568-0.5515$ \\
\hline 9 & 0.1025 & 2 & $0.4548-0.5550$ \\
\hline 10 & 0.1039 & 2 & $0.4544-0.5564$ \\
\hline 11 & 0.1039 & 2 & $0.4542-0.5564$ \\
\hline 12 & 0.1039 & 2 & $0.4542-0.5564$ \\
\hline
\end{tabular}

The objective of the remaining part of this work is to check the correlation between the width of the PBG and the indices ratio $n_{\mathrm{H}} / n_{\mathrm{L}}$, with $n_{\mathrm{H}}$ and $n_{\mathrm{L}}$ representing the high and the low refractive indices respectively. We studied the reflection of the one-dimensional Rudin-Shapiro photonic structure as a function of the index contrast. The index contrast or the optical contrast is defined as the ratio between the high and low refractive indices of the layers constituting the structure. For a one-dimensional structure, the index contrast is given by [14]:

$$
\delta=n_{H} / n_{L}
$$

Figures 7(a) and (c) show the optical reflection of a multilayer structure based on the Rudin-Shapiro model for the $5^{\text {th }}$ and $6^{\text {th }}$ iterations respectively, as a function of the index contrast and wavelength. Figures 7(b) and (d) illustrate the maps of the PBGs for the $5^{\text {th }}$ and $6^{\text {th }}$ iterations, respectively, where the blue zone represents the forbidden bands and the white zone the permissible ones. We observe that for the $5^{\text {th }}$ iteration, the first PBG appears with index contrast $\delta \approx 1.774$ and the width and number of PBGs increase considerably with the index contrast. Moreover, from Table V, we notice that the difference between the middle of the PGB $\left(\lambda_{\mathrm{m}}\right)$ and the reference wavelength $\left(\lambda_{0}=0.5\right)$ increases with the increase in the index contrast.

(a)
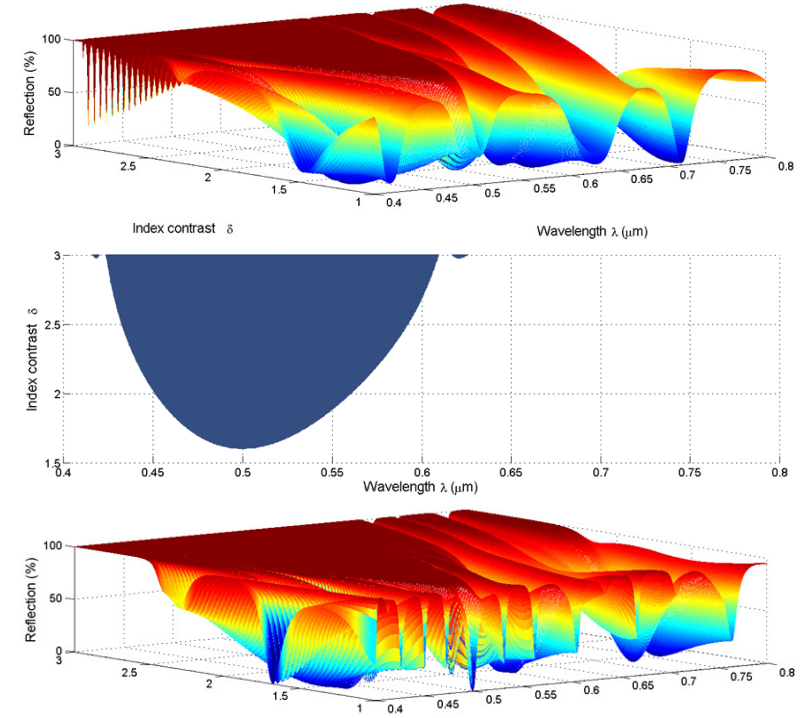

Index contrast

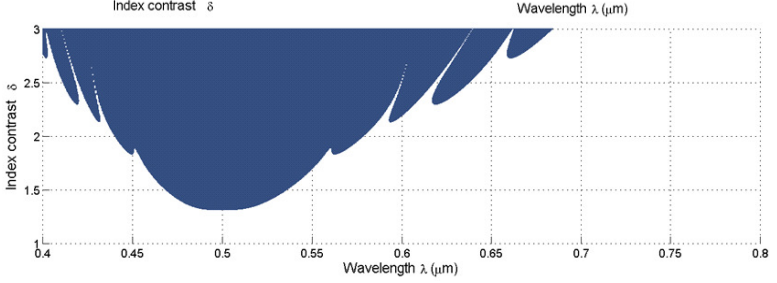

Fig. 7. Spectral aspect as a function of the contrast variation for the Rudin-Shapiro distribution. (a), (c) Variation of reflection spectra as a function of contrast and wavelength for the $5^{\text {th }}$ and $6^{\text {th }}$ iterations respectively. (b), (d) PBGs maps as a function of contrast and wavelength for the $5^{\text {th }}$ and $6^{\text {th }}$ iterations respectively

TABLE V. VARIATION OF THE PBGS WIDTH AND POSITIONS ACCORDING TO THE INDEX CONTRAST

\begin{tabular}{|c|c|c|c|c|c|c|c|c|}
\hline \multirow{2}{*}{$\boldsymbol{\delta}=\boldsymbol{n}_{\boldsymbol{H}} / \boldsymbol{n}_{\boldsymbol{L}}$} & \multicolumn{5}{|c|}{ 5th Iteration } & \multicolumn{4}{c|}{ 6th Iteration } \\
\cline { 2 - 9 } & $\lambda_{\max }$ & $\lambda_{\min }$ & $\lambda_{\boldsymbol{m}}$ & $\boldsymbol{\Delta} \boldsymbol{\lambda}_{\boldsymbol{B P G}}$ & $\lambda_{\max }$ & $\lambda_{\min }$ & $\lambda_{\boldsymbol{m}}$ & $\boldsymbol{\Delta} \boldsymbol{\lambda}_{\boldsymbol{B P G}}$ \\
\hline 1,6 & - & - & - & - & 0,4625 & 0,5437 & 0,5031 & 0,0812 \\
\hline 1,9 & 0,4581 & 0,5506 & 0,5044 & 0,0925 & 0,4452 & 0,5718 & 0,5085 & 0,1266 \\
\hline 2,2 & 0,4420 & 0,5746 & 0,5083 & 0,1326 & 0,4344 & 0,5898 & 0,5121 & 0,1554 \\
\hline 2,5 & 0,4332 & 0,5922 & 0,5127 & 0,1590 & 0,4280 & 0,5998 & 0,5139 & 0,1718 \\
\hline 2,8 & 0,4260 & 0,6038 & 0,5149 & 0,1778 & 0,4044 & 0,6310 & 0,5177 & 0,2266 \\
\hline
\end{tabular}

\section{CONCLUSION}

Based on the simulation of the photometric response of the Rudin-Shapiro multilayer structures as a function of the iteration $k$ we found that the number of PGBs and their widths increase with $k$ due to the increase of the number of layers from one iteration to another, which results in the generation of multiple internal reflections in the structure giving rise to more constructive interferences. The latter prevents certain wavelengths from propagating in the system in order to increase the width of the PBG. Additionally, the influence of the index contrast on the photonic band gap was investigated. It was found that the width of the PBG is proportional to the 
index contrast. Moreover, the number of PGBs increases considerably and their position shifts according to the index ratio so that the medium becomes more and more inhibiting for the propagation of light. Indeed, the increase of the index contrast generates more internal reflections on the interfaces of the high refractive indices' layers.

\section{ACKNOWLEDGMENT}

We would like to acknowledge the research deanship of University of Hail for the funding under the project RG191250.

\section{REFERENCES}

[1] K. Yasumoto, Electromagnetic theory and applications for photonic crystals, CRC Press, 2006

[2] M. Johri, Y. A. Ahmed, T. Bezboruah, "Photonic band gap materials Technology, applications and challenges", Current Science, Vol. 92, No. 10, pp. 1361-1366, 2007

[3] P. K. Bhattacharya, "Photonic crystal devices", Journal of Physics D: Applied Physics, Vol. 40, No. 9, 2007

[4] S. Hong-Yi, L. Zhi-Yean, G. Ben-Yuan, "Defect modes in multipleconstituent one-dimensional photonic crystals examined by an analytic Bloch-Mode approach", Chinese Physics Letters, Vol. 22, No. 2, pp 365-368, 2005

[5] A. H. Aly, "Electromagnetic propagation characteristics in one dimensional photonic crystal", in Wave Propagation in Materials for Modern Applications, InTech, pp. 193-200, 2011

[6] W. A. Goddard, Handbook of nanoscience, engineering, and technology, CRC Press, 2003

[7] I. Iliev, M. Nedelchev, E. Markov, "A novel 2D Z-shaped electromagnetic bandgap structure", Engineering, Technology \& Applied Science Research, Vol. 5, No. 1, pp. 760-763, 2015

[8] D. Schechtman, I. Blech, D. Gratias, J. W. Cahn, "Metallic phase with long-range orientational order and no translational symmetry", Physical Review Letters, Vol. 53, No. 20, Article ID 1951, 1984

[9] A. Bousselmi, A. Gharsallah, T. P. Vuong, "A novel high-gain quadband antenna with AMC metasurface for satellite positioning systems", Engineering, Technology \& Applied Science Research, Vol. 9, No. 5, pp. 4581-4585, 2019

[10] M. Rattier, H. Benisty, C. J. M. Smith, A. Bernaud, D. Cassagne, C. Jouanin, T. F. Krauss, C. Weisbuch, "Performance of waveguide-based two-dimensional photonic-crystal mirrors studied with Fabry-Perot resonators", IEEE Journal of Quantum Electronics, Vol. 37, No. 2, pp. 237-243, 2001

[11] J. H. Kim, L. Chrostowski, "Fabry-Perot cavity design in AlGaAs/GaAs using a photonic crystal slab for a resonant cavity detector", 19th Annual Meeting of the IEEE Lasers and Electro-Optics Society, Montreal, Canada, October 29-November 2, 2006

[12] J. P. Allouche, P. Liardet, "Generalized Rudin-Shapiro sequences", Acta Arithmethmetica, Vol. 60, No. 1, pp. 1-27, 1991

[13] V. Agarwal, M. E. Mora-Ramos, B. Alvarado-Tenorio, "Optical properties of multilayered Period-Doubling and Rudin-Shapiro porous silicon dielectric heterostructures", Photonics and NanostructuresFundamentals and Applications, Vol. 7, No. 2, pp. 63-68, 2008

[14] W. Rudin, "Some theorems on Fourier coefficients", Proceedings of the American Mathematical Society, Vol. 10, No. 6, pp. 855-859, 1959

[15] H. S. Shapiro, Extremal problems for polynomials and power series, MSc Thesis, Massachussetts Institute of Technology, 1951

[16] Z. Y. Li, "Principles of the plane-wave transfer-matrix method for photonic crystals", Science and Technology of Advanced Materials, Vol. 6, No. 7, pp. 837-841, 2005

[17] Y. Zeng, Y. Fu, X. Chen, W. Lu, H. Agren, "Extended planewave-based transfer-matrix approach to simulating dispersive photonic crystals", Solid State Communications, Vol. 139, No. 7, pp. 328-333, 2006
[18] A. Mouldi, M. Kanzari, "Design of microwave devices exploiting Fibonacci and hybrid periodic/Fibonacci one dimensional photonic crystals", Progress In Electromagnetics Research B, Vol. 40, pp. $221-$ 240, 2012

[19] K. Ben Abdelaziz, J. Zaghdoudi, M. Kanzari, B. Rezig, "A broad omnidirectional reflection band obtained from deformed Fibonacci quasi-periodic one dimensional photonic crystals", Journal of Optics A: Pure and Applied Optics, Vol. 7, pp. 544-549, 2005 